\title{
Ambivalence du calculable et crise du jugement
}

jean.lassegue@ehess.fr

Résumé

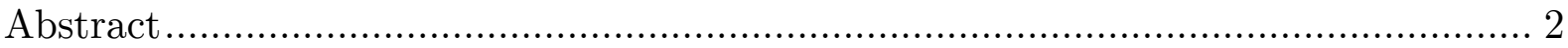

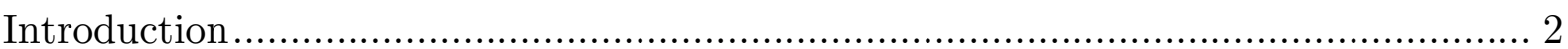

1. Intérêt de la notion de calculable pour la philosophie du droit actuel.................... 3

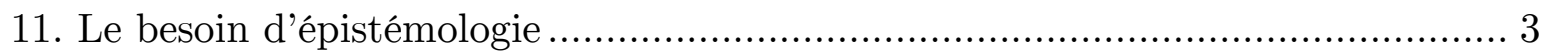

12. L'éthique du déterminisme et de la prédictibilité.................................... 5

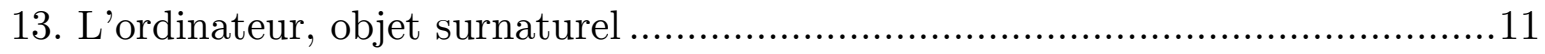

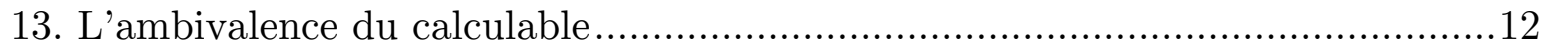

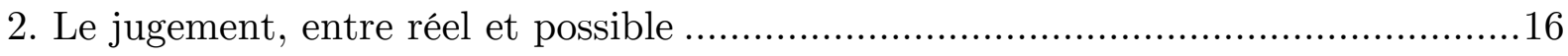

21. Leibniz au pays des Big data ................................................................. 16

22. L'argument du Dominateur et la réalité spatio-temporelle du jugement ..........20

Le jugement, à la croisée du calculable et de l'incalculable ....................................24

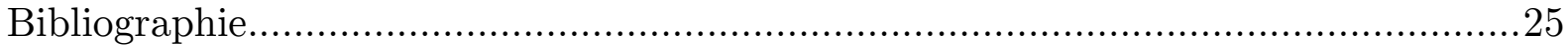

\section{Résumé}

L'article traite de l'informatisation du droit qui met en crise l'exercice du jugement en le privant d'une partie de ses prérogatives fondamentales. Il explore le rapport entre deux sortes de légitimité, juridique et numérique, en s'appuyant sur des arguments tirés de l'histoire des sciences: l'argument du Dominateur de Diodore Cronos, la métaphysique alphabétique de Leibniz et le théorème de limitation du calculable de Turing. 


\section{Mots-clés}

Digitalisation du droit, Calculable, Déterminisme, Prédictibilité, Big Data, réalité spatio-temporelle du jugement, Diodore Cronos, Leibniz, Turing.

\section{Abstract}

The article deals with the digitalization of law which disturbs the exercise of judgement by partially depriving it from its basic prerogatives. It explores the relationship between two types of legitimacy, juridical and digital, by relying on arguments from the history of science: the master argument by Diodorus Cronus, the alphabetical metaphysics of Leibniz and the theorem of limitation in computability by Turing.

\section{Keywords :}

Digitalisation of law, Computable, Determinism, Predictibility, Big Data, spatiotemporal reality of judgement, Diodorus Cronus, Leibniz, Turing.

\section{Introduction}

Le but de cet article est de ressaisir sous un angle épistémologique les débats actuels sur la digitalisation du droit et de la justice pour essayer de prendre la mesure des transformations qui, dans ce domaine, mettent aujourd'hui en crise la notion de jugement. Cette crise peut être décrite d'un mot : c'est la délégation aux machines qui semble priver les humains d'une de leurs prérogatives les plus fondamentales, à savoir la capacité de produire un jugement de façon autonome. La notion actuelle de calcul et ses conséquences dans la sphère du droit constituent donc le centre de gravité des pages qui suivent. Historiquement, on montrera que le projet de mécanisation du jugement s'est constitué dès l'âge classique mais que ce sont les limitations internes de 
la calculabilité telles qu'elles ont été mises au jour dans les années 30 du siècle dernier qui ont, de façon paradoxale au premier abord, renforcé à l'extrême la tendance à la mécanisation, aggravant la crise qui affecte la notion de jugement en droit. On montrera cependant que ce moment de crise a l'avantage de révéler le cadre collectif nécessaire à l'institution de l'instance juridique de jugement. S'il y a crise du jugement, il y a donc aussi moyen d'y pallier et de renouveler de l'intérieur le régime même de la légalité juridique.

\section{Intérêt de la notion de calculable pour la philosophie du droit actuel}

Pour réussir à envisager la situation propre à la digitalisation du droit et de la justice contemporaine dans un cadre tel qu'une évaluation philosophique devienne possible, il nous paraît nécessaire de revenir au cœur épistémologique de cette digitalisation, à savoir le concept de calculabilité. Il nous faut néanmoins commencer par justifier ce retour à l'épistémologie en répondant à deux objections préalables qui visent, à l'opposé, à tenter de séparer l'aspect épistémologique et l'aspect juridique de la notion de justice digitale. Pour nous, c'est précisément cette séparation qui est l'indice d'une crise dans la notion de jugement et c'est donc elle dont il faut d'abord commencer par prendre la mesure.

\section{Le besoin d'épistémologie}

Une première objection consiste à dire que l'on n'a pas besoin de connaître les arcanes du concept logico-mathématique de calculabilité pour analyser les effets sociaux d'un tel concept sur le droit et la justice et que l'on peut donc sans dommage s'en tenir à ce seul domaine, déjà suffisamment vaste en lui-même quand on voit la multitude des changements opérés sur le droit par l'introduction de la digitalisation (dans le droit de la concurrence, le droit de la propriété intellectuelle, la fiscalité, l'arbitrage, le procès, etc.). Cependant, cette objection, parce qu'elle suppose une dichotomie a priori entre le niveau conceptuel dont on est censé pouvoir faire l'économie et le niveau proprement 
social du droit est elle-même sujette à caution : sans chercher à réduire le conceptuel au social, ce sont au contraire les modalités sans cesse à renégocier de leur rapport qui permettent, selon nous, une évaluation philosophique de la situation juridique contemporaine. Une étape épistémologique ayant le concept de calculabilité pour objet est donc souhaitable dans l'analyse du droit et de la justice à l'ère digitale.

Une deuxième objection consiste à défendre l'idée selon laquelle invoquer le concept de calculabilité dans le cas du droit et de la justice revient à tailler un costume trop grand pour eux car l'ère digitale touche également bien d'autres domaines, de la cartographie à la finance en passant par les transports et le cinéma, et ne vise donc pas prioritairement le domaine juridique. Là encore, il s'agirait de dissocier le droit et la justice de l'épistémologie de la calculabilité en laissant entendre que leur digitalisation ne requiert pas d'analyse proprement épistémologique préalable, sauf à l'envisager d'un point de vue très général comme un cas parmi beaucoup d'autres. Il est également possible de répondre à cette objection et de préciser du même coup notre projet. Le domaine du droit et celui de la justice possèdent un objet spécifique qui les distingue de tous les autres domaines : la perpétuelle redéfinition de la norme sociale. Or c'est précisément du point de vue de la redéfinition de la norme en général que se situe le rapprochement philosophique possible avec l'épistémologie du concept de calculabilité : nous envisagerons en effet le concept de calculabilité du point de vue de la norme qu'il cherche à instituer en rupture avec le droit traditionnel et non des prouesses techniques que son incarnation informatique permet de réaliser. Quelle est cette norme ?

Bien que cela soit tout d'abord contre-intuitif dans le cas d'un concept de nature mathématique, le concept de calculabilité tel qu'il a été mis en lumière dans les années trente du siècle dernier, en particulier par Turing ${ }^{1}$, enveloppe une injonction, celle, pour résoudre un problème quelconque, de se mettre à la place de ce qu'exécute une

\footnotetext{
1 A. M. Turing, "On Computable Numbers with an Application to the Entscheidungsproblem", Proceedings of the London Mathematical Society, Volumes 2-42, Issue 1, 1 January 1937: 230-265.
} 
machine effectuant un calcul sans signification (un algorithme). Turing distingue radicalement le niveau des marques graphiques dont la combinaison s'opère en suivant des règles automatiques qui ne requièrent aucune intelligence d'une part et le résultat de cette combinaison que le calculateur humain interprète comme porteur de signification de l'autre. L'injonction contenue dans le concept de calculabilité issue de ce dualisme devenu radical ${ }^{2}$ est donc bien l'expression d'une norme que l'on pourrait appeler l' « injonction de la réduction au mécanique »- le mécanique étant entendu ici comme combinatoire strictement graphique ${ }^{3}$. Aussi, contrairement aux apparences, ce point de vue normatif qui se trouve au fondement du concept de calculabilité a-t-il un sens social et son épistémologie se situe-t-elle de plain-pied avec la réflexion sur ce que la norme juridique tente d'instituer. Concevoir un rapport entre épistémologie de la calculabilité et théorie juridique de la norme a donc philosophiquement un intérêt parce qu'en situant la crise du jugement dans la compétition entre les deux types de norme, il devient aussi possible d'envisager comment surmonter le problème.

Une fois ces objections levées et le rapport - encore à préciser - entre norme du calcul et norme juridique envisagé, il nous faut décrire plus avant ce que l'on pourrait appeler le « sens social »du concept de calculabilité pour rendre possible l'analyse des rapports qu'il entretient avec la norme juridique.

\section{L'éthique du déterminisme et de la prédictibilité}

Nous reprenons ici à notre compte l'expression d'《éthique » associée à des notions épistémologiques, comme a pu le faire David R. Lachterman dans The Ethics of Geometry quand il tentait de replacer la pratique mathématique antique et moderne

\footnotetext{
${ }^{2}$ Historiquement, outre ses bases métaphysiques, ce dualisme est le fruit de l'idée de formalisation telle qu'elle a été progressivement mise en place dans les travaux de Hilbert à partir du début du $20^{\text {ème }}$ siècle. Cf. A. Garapon et J. Lassègue, Justice digitale, Paris, Presses universitaires de France 2018.

3 Sur ce point J. Lassègue \& G. Longo, "What is Turing's Comparison between Mechanism and Writing Worth?" dans S.B. Cooper, A. Dawar, and B. Löwe (Eds.), How the World Computes, Lecture Notes in Computer Science 7318, Springer: 451-462.
} 
dans un cadre culturel souterrain qui en gouverne l'interprétation ${ }^{4}$. Il faut, pour comprendre la nature du rapport entre les deux types de norme, de calcul et de droit, remonter à la source de la notion de calcul dans la science classique et au fait que la nature apparaît, à partir du $17^{\text {ème }}$ siècle, comme le lieu d'exercice d'une volonté humaine conçue comme infinie.

Le jeu se joue à trois : Dieu, la nature et la volonté humaine. C'est en particulier le cas chez Descartes pour lequel l'infinité de la volonté dérivant de Dieu ${ }^{5}$ est couplée à la maîtrise mathématique de la nature et en particulier des organismes ${ }^{6}$. La connaissance de la nature passe par l'exploration d'un lieu homogène, celui de l'espace idéalisé dans lequel les droites n'ont pas d'épaisseur comme c'était déjà le cas chez Euclide $^{7}$ mais aussi dans lequel les objets se déplacent sans frottement. Le moteur de cette exploration réside dans le caractère infini de la volonté humaine qui est susceptible de se manifester via un usage de signes particuliers, les signes mathématiques, comme le fait remarquer Galilée ${ }^{8}$. La volonté infinie s'incarne ainsi dans des signes qui rendent possible sa manifestation. « Mathématique » est donc l'adjectif qui désigne un double mouvement : il caractérise la vérité du rapport entre Dieu et la nature d'une part et

\footnotetext{
4 David. R. Lachterman, The Ethics of Geometry: a Genealogy of Modernity, Routledge, Londres, 1989 où il montre en particulier que c'est la différence entre contemplation des objets mathématiques et construction de ces mêmes objets qui distingue radicalement l'antiquité et le monde moderne.

5 « Il n'y a que la seule volonté, que j'expérimente en moi être si grande, que je ne conçois point l'idée d'aucune autre plus ample et plus étendue : en sorte que c'est elle principalement qui me fait connaître que je porte l'image et la ressemblance de Dieu. » Descartes, Méditation IV ; AT IX-1, p. 46.

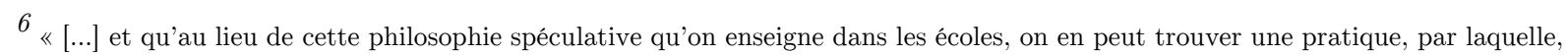
connaissant la force et les actions du feu, de l'eau, de l'air, des astres, des cieux, et de tous les autres corps qui nous environnent, aussi distinctement que nous connaissons les divers métiers de nos artisans, nous les pourrions employer en même façon à tous les usages auxquels ils sont propres, et ainsi nous rendre comme maîtres et possesseurs de la nature. Ce qui n'est pas seulement à désirer pour l'invention d'une infinité d'artifices, qui feraient qu'on jouirait sans aucune peine des fruits de la terre et de toutes les commodités qui s'y trouvent, mais principalement aussi pour la conservation de la santé, laquelle est sans doute le premier bien et le fondement de tous les autres biens de cette vie [...]». Descartes, Discours de la méthode, VI ; AT VI 61-62.

${ }^{7}$ Cf. Giuseppe Longo, Maël Montévil et Arnaud Pocheville, "L'incompressible complexité du réel et la construction évolutive du simple", dans Complexité-Simplexité, A. Berthoz et J.-L. Petit eds, Paris, Collège de France, 2014, § 39.

${ }^{8}$ Galilée, L'essayeur, § 50 : « La philosophie est écrite dans cet immense livre qui continuellement reste ouvert devant les yeux (ce livre qui est l'Univers), mais on ne peut le comprendre si, d'abord, on ne s'exerce pas à en connaître la langue et les caractères dans lesquels il est écrit. II est écrit dans une langue mathématique, et les caractères en sont les triangles, les cercles, et d'autres figures géométriques, sans lesquelles il est impossible humainement d'en saisir le moindre mot ; sans ces moyens, on risque de s'égarer dans un labyrinthe obscur. » Ces phrases si connues n'en sont pas moins profondément énigmatiques quand on remarque que la philosophie se donne à même des signes eux-mêmes conçue comme exprimant la nature.
} 
désigne la possibilité d'une extension volontaire de ce rapport via des signes particuliers, ceux de la mathématique, à des domaines naturels encore inexplorés d'autre part. En ce sens, l'adjectif « mathématique » possède bien la nature duelle d'un signe qui, à la fois, « renvoie à » tout en recherchant à asseoir sa propre manifestation comme expression de la volonté. Le signe mathématique devient ainsi déchiffrable à la fois dans la nature par le biais du calcul et dans le mouvement de découverte de lui-même comme volonté. L'expansion des signes mathématiques à des domaines toujours nouveaux est donc le signe même qu'une volonté y est à l'œuvre. Calcul et volonté sont ainsi les deux faces d'une même médaille.

Un chapitre de l'histoire de l'idée de fonction mathématique à l'époque moderne est de ce point de vue un bon exemple du mouvement d'expansion et de conquête progressive de l'infini mathématique conçu comme volonté partie à la recherche d'ellemême par le biais de signes : de l'abus de langage de Descartes qui, dans la Géométrie, appelle « courbes mécaniques » des courbes dont il n'a pourtant pas encore les moyens mathématiques de tracer tous les points ${ }^{9}$ à l'apparition de l'algorithme du calcul infinitésimal chez Leibniz, la science classique cristallise vers les années 70 la possibilité d'une prédiction déterministe des événements de la nature ${ }^{10}$ et des décisions juridiques ${ }^{11}$ au moyen de signes mathématiques qui manifestent l'infini comme volonté : le signe mathématique comme rapport de signification et comme auto-affirmation de $\mathrm{soi}^{12}$ culmine dès lors dans l'idée de calcul ${ }^{13}$.

\footnotetext{
${ }^{9}$ Descartes reconnaît par existence l'existence de la courbe logarithme (lettre à Mersenne du 12 Septembre 1638), à partir du tracé de certains de ses points par la machine à équerres mobiles qu'il utilise dans la Géométrie, mais il est incapable de justifier mathématiquement dans une mesure euclidienne ce qu'il est cependant capable de reconnaître intuitivement.

10 Leibniz, "Nova Methodus pro Maximis et Minimis" (1674 publié en 1684).

11 Leibniz, "De Justitia et Novo Codice" (1678), Grua II, p. 621-622. Cf. Mate Paksy, "Leibniz et les questions de l'ontologie juridique : la science, les règles et le concept du droit", Asterion, (2018).

12 La phrase également si connue de Leibniz ("Dialogus", die Philosophischen Schriften, Gerhardt ed., tome VII, p. 191) : «Pendant que Dieu calcule et qu'il exerce son esprit, le mode se fait » montre bien que le caractère duel du mathématique, à la fois calcul et cogitation, est un rapport de sémiose avant d'être un rapport de causalité puisqu'il n'y a qu'un rapport de concomitance et non de causalité entre l'action opérée par Dieu et la constitution de la nature.

13 Comme le fait remarquer Couturat à propos de Leibniz : «La nature est le produit d'une logique divine, de ce calcul immense qui est la création ; elle est pour nous une admirable machine à calculer, car elle nous fournit, tout faits, les résultats de calculs qui dépassent la portée de notre entendement». Couturat, La logique de Leibniz, Alcan, Paris, 1901, p. 256.
} 
Si l'on quitte la science classique et que l'on s'interroge sur la façon dont l'idée de calcul a été ressaisie à partir des années 30 du siècle dernier qui voient la naissance de la théorie actuelle du calcul, on ne peut pas ne pas être frappé par la similitude des points de départ ${ }^{14}$ même s'il ne s'est pas trouvé de philosophe à l'époque contemporaine qui fût à la fois mathématicien, physicien et juriste pour conserver telle quelle la cohérence du point de vue leibnizien ${ }^{15}$. C'est en effet dans le cadre d'un paradigme déterministe des plus classiques que la question de la nature du calcul se pose, à savoir le « programme de Hilbert » qui vise à étendre le déterminisme prédictif tel qu'il avait été envisagé en physique depuis l'âge classique à la déduction des théorèmes au moyen du calcul dans le cadre d'une axiomatique formalisant les énoncés mathématiques. De ce point de vue, comme l'a remarqué G. Longo ${ }^{16}$, Hilbert construit une analogie entre la déduction des théorèmes dans une axiomatique formalisée et la détermination des événements dans la nature. Mais de même que dans les sciences de la nature où l'association entre calcul et déterminisme prédictif avait été remise en cause à l'occasion des travaux portant sur les processus irréversibles de la thermodynamique au $19^{\text {ème }}$ siècle ou dans les théories de type critique telles qu'elles apparaissent dans les travaux de Poincaré en mécanique céleste au début du $20^{\text {ème }}$, de même l'association du calcul et de la déduction de théorèmes dans le cadre de l'axiomatique formalisée fut remise en question, de l'intérieur même de la perspective formaliste, par des limitations internes qui concernent notamment l'idée de calcul.

\footnotetext{
14 Nous suivons ici G. Longo, "Au sujet des lois dans les sciences humaines et de la nature" dans Loi des hommes des dieux et de la nature ; éléments pour une analyse transversale, Spartacus-IDH, Paris, 2017, p. 5 : « Il est très à la mode aujourd'hui, à l'époque des ordinateurs, d'identifier algorithme (règle formelle à appliquer mécaniquement) et loi de la nature [...]. On fait alors un pas en arrière : l'algorithme est normatif pour la machine, il norme ses calculs, comme la loi de Dieu règle toute trajectoire. [...]. Encore une fois, le mythe de l'Univers-ordinateur (le génome, l'évolution, le cerveau... tous gouvernés par des algorithmes) revient à une métaphysique et à une notion de détermination qui précède la science du XIXe siècle [...]. »

${ }^{15}$ La théorie du droit semble en effet faire sécession et le positivisme d'un Kelsen ne s'intègre pas, à un système englobant science mathématique, sciences de la nature et science juridique.

${ }^{16}$ Cf. F. Bailly et G. Longo, Mathématiques et sciences de la nature ; la singularité physique du vivant, Hermann, Paris, 2007.
} 
Si l'on observe en effet l'article fondateur de la théorie de la calculabilité rédigé par Turing en 1936, on remarque qu'il porte sur le rapport entre deux concepts : celui de déterminisme et celui de prédictibilité.

Déterminisme tout d'abord. L'exemple de calcul sur lequel porte son analyse dans l'article de 1936 est celui de l'expansion décimale infinie d'un nombre réel ${ }^{17}$. Arrêtonsnous un instant sur cet exemple. On y définit un domaine numérique particulier, celui des nombres réels pouvant être calculés par une procédure systématique, c'est-à-dire par un ensemble fini de règles : les réels dits « calculables ». Ainsi l'expansion décimale d'un nombre réel quelconque est-elle infinie mais la méthode de calcul (l'algorithme) pour égrener indéfiniment cette expansion est, elle, finie (c'est ainsi que nous avons appris à extraire les racines carrées ou les décimales de pi). Le cas certes particulier de l'expansion décimale infinie d'un nombre réel envisagé par Turing doit cependant nous rappeler que l'intervention de l'infini a aussi une dimension normative, si par norme on entend l'expansion du domaine de la volonté par le biais des signes mathématiques. La constitution par Turing d'un modèle du calcul fondé sur une machine abstraite appelée depuis «machine de Turing » a donc pour moteur une volonté infinie opérant de façon strictement déterministe. La machine qui calcule l'expansion décimale infinie d'un nombre réel est donc en tout point semblable à l'idéal poursuivi par la science classique et on comprend qu'elle incarne à elle seule ce que nous avons appelé plus haut l'« injonction de la réduction au mécanique ». Les nombres réels «calculables » sont ainsi les nombres réels dont on a pu montrer qu'ils obéissaient à l'injonction de la réduction au mécanique même si leur expansion décimale est infinie.

Prédictibilité ensuite. Contrairement à l'idéal de la science classique revisité par Hilbert, Turing montre que cette injonction ne peut pas être obéie dans tous les cas si l'on s'en tient exclusivement au calcul tel qu'il est exécuté par la machine puisque, en

\footnotetext{
17 A. M. Turing, "On Computable Numbers with an Application to the Entscheidungsproblem", Proceedings of the London Mathematical Society, p. 230. La première phrase de l'article est la suivante : « Les nombres "calculables" peuvent être décrits succinctement comme les nombres réels qui, exprimés par nombre décimal, sont calculables par des moyens finis. »
} 
héritier indocile de la problématique de Hilbert, il parvient à démontrer, sur un problème particulier qu'il exhibe ${ }^{18}$, les limitations concernant la prédictibilité d'un résultat inhérentes à toute démarche strictement calculatoire. On n'a pas assez remarqué que le problème qui démontre l'existence d'une limitation interne au calcul tel qu'il est posé par Turing porte précisément sur une question de prédictibilité : ce que montre le problème imaginé par Turing, c'est l'incapacité d'une machine A à prévoir le comportement futur d'une autre machine B (un comportement comme celui consistant à produire une expansion décimale) par la seule inspection mécanique de l'ensemble fini des règles de B. Turing démontre qu'il n'est pas possible de savoir à l'avance si la machine B inspectée par la machine A continuera ou pas à produire indéfiniment l'expansion décimale (ou tout autre résultat) pour lequel elle a été paramétrée. Le problème que Turing exhibe va donc directement contre l'idéal classique d'un rapport d'implication entre calcul et prédictibilité : Turing démontre ainsi qu'il faut découpler calcul et prédictibilité dans le cadre du « programme de Hilbert » de même que Poincaré avait montré en son temps que calcul et prédictibilité devaient être découplées en mécanique céleste.

On se rappelle que ce rapport était fondé, à l'âge classique, sur le postulat de l'expansion d'une volonté infinie via les signes mathématiques et d'un rapport univoque entre un Dieu conçu comme fondement du sens et la nature conçue comme se rapportant à lui comme à sa source. Dans le cas de Turing, tout se passe au contraire comme si la volonté continuait bien d'être infinie (dans la manifestation de l'expansion décimale indéfinie des nombres réels par exemple) mais qu'il n'était pas possible de savoir à l'avance si elle l'était réellement ou pas dans son comportement ultime. Deux choses sont ainsi connues quant à l'injonction de la réduction au mécanique : d'une

\footnotetext{
18 Contrairement à une habitude solidement ancrée, ce qu'il est convenu d'appeler le « problème de l'arrêt » n'est pas explicitement énoncé dans l'article de Turing puisqu'il n'apparaît que plus tard, chez Martin Davis en 1958. En revanche, le problème exhibé par Turing est bien celui d'une machine qui continue indéfiniment son calcul et dont on ne peut pas savoir à l'avance quel sera son comportement ultime.
} 
part elle ne peut pas être intégrale, d'autre part il n'est pas possible de savoir à l'avance si elle sera ou non illimitée. Mais la conséquence d'un tel état de fait est à première vue paradoxale parce que loin de limiter l'injonction du recours au calcul, elle exige au contraire son vérification continuelle. On doit donc dire que cette recherche de confirmation se nourrit donc de sa propre limitation. La calculabilité institue de ce fait un champ dont l'extension se nourrit de ce qui lui échappe : c'est la raison de l'expansion vertigineuse du calculable dont on voit aujourd'hui les effets sociétaux. Il y a là une transformation interne de l'idéal de la science classique qui, loin d'être un simple abandon de celui-ci, institue désormais une nouvelle figure de la volonté dont il n'est plus certain qu'elle soit toujours en état d'établir une mesure entre fini et infini dans sa double composante de rapport à la nature et d'auto-affirmation de soi.

\section{L'ordinateur, objet surnaturel}

L'ordinateur en tant que machine physique est l'incarnation matérielle de la machine mathématique de Turing. Il est le lointain descendant du dualisme du corps et de l'âme cher à l'âge classique dans la mesure où il est fondé sur la séparation radicale entre le niveau du matériel («hardware ») et du logiciel (« software »). Mais il faut bien noter que la notion de « machine » se laisse alors appréhender à fronts renversés. En tant que machine physique, l'ordinateur est à proprement parler un objet surnaturel car ses opérations obéissent à un déterminisme prédictif strict contrairement à ce qui se produit dans la nature physique comme on le sait depuis Poincaré, déterminisme prédictif qui est artificiellement maintenu dans l'ordinateur par ce que l'on peut appeler à bon droit un miracle technologique ${ }^{19}$. Or, en tant que machine abstraite, la machine produit un résultat paradoxal puisque c'est en s'en tenant à des moyens strictement calculatoires qu'on démontre par un raisonnement par l'absurde le nécessaire découplage entre déterminisme calculatoire et prédictibilité. Il s'agit donc

\footnotetext{
19 G. Longo, "Laplace, Turing et la géométrie impossible du « jeu de l'imitation »: aléas, déterminisme et programmes dans le test de Turing", Intellectica, n. 35, 2002/2, pp. 131-162.
} 
bien de fronts renversés puisqu'on s'attendrait à se trouver dans la situation habituelle consistant à se heurter à des limitations empiriques sur le plan de la matière physique (concernant par exemple le degré limité de précision dans la mesure physique) et une absence de limitation sur le plan conceptuel: or c'est l'inverse qui se produit. L'ambivalence de la notion de calculable peut donc se résumer à ceci : le déterminisme de l'ordinateur est artificiellement maintenu dans le monde physique tandis qu'il ne l'est pas en tant que machine mathématique puisqu'on démontre à ce niveau le découplage du calcul déterministe et de la prédictibilité par le biais d'un théorème de limitation.

L'ordinateur comme machine matérielle apparaît ainsi comme l'incarnation de l'expansion généralisée du calcul déterministe mais ne manifeste pas en lui-même la condition de non-calculabilité qui le rend néanmoins possible sur le plan conceptuel. La limite qui restreint a priori l'expansion du calculable passe donc à peu près inaperçue puisqu'au niveau matériel de l'ordinateur, elle se limite à la confirmation continuelle de la calculabilité des problèmes rencontrés empiriquement. C'est ce à quoi nous assistons aujourd'hui dans l'injonction toujours plus clairement affichée d'une réduction au mécanique.

Cette situation a évidemment des conséquences sur le statut de la norme : c'est dorénavant le rapport calculable / non-calculable qui en constitue le fondement et qui opère une scission interne au concept de volonté qui jusque-là se présentait dans son unité comme infini. C'est cette scission interne au concept de volonté dont il va falloir étudier les conséquences sur la notion de jugement.

\section{L'ambivalence du calculable}

Le résultat d'une telle analyse permet de définir deux axes propres au concept de calculabilité : d'une part un axe « horizontal » pourrait-on dire dans lequel le calcul peut s'étendre sans limites à des domaines toujours plus étendus en vue de corroborer leur calculabilité au moyen de l'ordinateur ; d'autre part, un axe « vertical » dans 
lequel le calcul est l'instrument de sa propre limitation interne. La difficulté de l'usage du concept de calculabilité consiste à tenir ensemble les deux axes en question, seule attitude qui soit à même de rendre compte de «l'ambivalence» du calculable mais aussi de la possibilité même du jugement.

S'en tenir au contraire à l'un ou l'autre axe clarifie deux attitudes contradictoires, d'une part celle des tenants du tout-calculable pour lesquels seul l'axe vertical devrait retenir l'attention ${ }^{20}$ et d'autre part celle de ceux qui voudraient sauver un domaine non-calculable en tentant par tous les moyens de circonscrire un domaine qui lui échapperait, domaine pourtant jusqu'à présent insaisissable. Ces deux attitudes sont d'ailleurs souvent réunies dans un même discours qui vante d'un seul mouvement les mérites de l'expansion illimitée du numérique tout en fustigeant son absence de limite. C'est ce que nous allons voir maintenant sur le cas de l'ambivalence à l'égard des transformations du droit liées au numérique.

Commençons par remarquer, même si ce n'est pas le lieu d'entrer ici dans les détails techniques de tel ou tel logiciel ou base de données juridique, que les grandes catégories fondatrices du droit sont brouillées par l'apparition du numérique. Principe de l'égalité devant la loi, distinction entre le droit et le fait ou entre le public et le privé, pour ne citer qu'eux, sont remis subrepticement en question. Qu'on en juge : les projets d'une adaptation de la loi aux cas individuels (selon l'âge, le niveau d'assurance, la situation particulière des lieux, etc.) dérogent au principe d'égalité devant la loi ; l'introduction massive de statistiques portant sur les décisions de justice telles qu'elles sont effectivement prises tend à déplacer la légalité de la sphère du droit à celle de la régularité des faits ; quant aux fonctions mêmes assumées par les différents participants aux procès, elles sont remises en question dès lors que la souveraineté de leur jugement

\footnotetext{
20 Une lecture hâtive de Turing lui prête cette attitude en faisant de lui son porte-parole le plus prestigieux via la constitution d'un test dit « de Turing ». Pour la critique de la notion de test de Turing et de sa finalité supposée, cf. J. Lassègue, "Turing, entre formel et forme ; remarques sur la convergence des perspectives morphologiques", Intellectica, 2002/2 (35): 185-198 qui commente l'article de G. Longo "Laplace, Turing et la géométrie impossible du « jeu de l'imitation »: aléas, déterminisme et programmes dans le test de Turing", paru dans le même numéro.
} 
peut faire l'objet d'une analyse statistique qui font apparaître les individus et leurs biais derrière leur fonction (qu'on songe par exemple aux évaluations statistiques des juges américains qui, tout en contribuant utilement à la clarification de leurs biais, tend également à diminuer leur autorité ou simplement leur liberté de juger autrement que la majorité de leurs collègues dans des affaires similaires). Ce brouillage des catégories fondatrices du droit nourrit une attitude ambiguë à l'égard du numérique qui provient de l'ambivalence du calculable décrite plus haut et qui se retrouve dans les différents groupes sociaux impliqués.

Dans le cas des justiciables peu au fait de la réalité des transformations numériques en cours et qui acceptent dorénavant comme une évidence l'idée d'une délégation du jugement à des logiciels de prise de décision juridique (ce que nous avons appelé plus haut l'« injonction de la réduction au mécanique »), l'ambivalence porte sur la nature de l'objectivité du jugement considérée soit négativement comme portant atteinte à l' « humanité » (sans plus de précision) qui doit pourtant guider la décision de justice soit positivement comme le moyen enfin trouvé de pallier les préjugés et les biais personnels des juges et d'atteindre à l'égalité devant la loi.

Dans le cas des juristes et des professionnels du droit, on remarque, même si la connaissance de la réalité et des limites des logiciels d'aide à la décision juridique est en général bien supérieure, une attitude analogue consistant soit à refuser catégoriquement le brouillage des catégories juridiques fondatrices et partant tout immixtion du numérique dans le juridique soit à envisager une réponse purement technique consistant à se lancer à corps perdu dans l'élaboration d'un nouveau contenu du droit : droit de la concurrence, droit de la propriété intellectuelle, fiscalité, etc. Les deux réactions passent à côté du problème de la constitution d'une forme nouvelle de légalité dans laquelle le numérique est dorénavant appelé à jouer un rôle de plus en plus marqué. 
De même, l'ambiguïté se retrouve en sociologie du droit où l'ambivalence du calculable est essentiellement conçue sur le mode d'un nouveau pouvoir : soit parce qu'on lui prête un rôle occulte soit parce que le codage numérique est lui-même conçu comme la cause des changements dans la sphère du droit. Dans le premier cas, en insistant sur l'autonomie de l'axe horizontal de l'expansion indéfinie du calculable par rapport à l'axe vertical de sa limitation interne, on est conduit à prêter au numérique un rôle de surveillance et de manipulation, ce dont la notion de « gouvernementalité algorithmique $»^{21}$ cherche à rendre compte. Dans le deuxième, en prêtant au codage le rôle d'une activité qui cause ${ }^{22}$ et non pas seulement qui autorise ${ }^{23}$, on se refuse à concevoir l'ambivalence du calcul comme la marque de sa nature sémiotique en identifiant la notion de cause et l'axe de la toute-puissance du calcul.

L'amphibologie des concepts de la réflexion concernant le cas du droit et de la justice numérique a donc pour origine une situation particulière dans laquelle l'ambivalence du numérique est difficilement conçue dans la globalité de ses deux axes. C'est ce qui provoque la crise du jugement qui se cristallise dans la question de savoir ce qu'il convient de déléguer aux machines sans crainte d'être relégué par elles. Que la solution à cette crise soit exclusivement recherchée dans la propension toujours plus grande à opérer une réduction au calcul laisse de côté le fait que cette tendance tire sa force de ce qui lui fait défaut - l'impossibilité de s'en tenir exclusivement au plan du seul calculable. Et c'est bien cette situation dont nous faisons l'expérience aujourd'hui avec ce qu’il est convenu d'appeler les données massives (« Big Data ») qui semble

\footnotetext{
21 A. Rouvroy \& B. Stiegler, « Le régime de vérité numérique », Socio, 4 | 2015, 113-140.

22 C'est par exemple le cas de Lawrence Lessig dans son article célèbre "Code is Law" (Harvard Magazine, 2000) : « Chaque âge possède son régulateur potentiel, sa façon de menacer la liberté [...]. Le nôtre est l'âge du cyberespace [...]. Ce régulateur est le code - le logiciel et le matériel qui fait du cyberespace ce qu'il est. Le code ou son architecture, définissent les termes selon lesquels la vie dans la cyberespace est vécue. Il détermine combien il est facile de protéger la vie privée ou combien il est facile de censurer la parole. Il détermine si l'accès à l'information est général ou si l'information est limitée à des zones. Il affecte qui voit quoi et qui est surveillé. On ne commence à apercevoir les nombreuses manières dont le cyberespace est règlementé que si l'on comprend la nature de ce code. »

23 Il s'agit ici d'une périphrase qui cherche à décrire la notion d' « enablement ». Cf. Giuseppe Longo, Maël Montévil. "Extended criticality, phase spaces and enablement in biology" $\S 7$ dans Chaos, Solitons and Fractals, Elsevier, 2013, Emergent Critical Brain Dynamics, 55, pp.64-79.
} 
corroborer l'omniprésence du numérique en masquant le manque sur lequel repose cette dynamique. Pourtant, cette situation conduit à une impasse logique qui ne fait que renforcer la nécessité d'une nouvelle approche du concept de volonté. C'est ce que nous allons voir maintenant.

\section{Le jugement, entre réel et possible}

Les attitudes à l'égard des transformations numériques du droit exigent de s'interroger sur ce qui définit aujourd'hui la légalité. Nous voudrions montrer maintenant que seule la prise en compte des deux axes de la calculabilité rend possible d'en expliciter la norme et partant, son rapport avec le droit.

\section{Leibniz au pays des Big data}

Si la tradition philosophique peut aider à clarifier le rapport entre norme calculatoire et norme juridique, un nom émerge tout particulièrement, celui de Leibniz, dont la philosophie résume à bien des égards toute l'ambition de ce qu'il est convenu d'appeler aujourd'hui les Big data, ces données informatiques que l'usage des ordinateurs connectés ont multiplié dans des proportions telles qu'elles mettent au défi toute compréhension de leur organisation. Dans le cas du droit, l'expansion généralisée de l'usage des données via l'informatisation de la société laisse en effet penser que la jurisprudence passée couvrirait un nombre de cas tel que l'avenir serait intégralement décidable au sens logique, c'est-à-dire que l'on aurait les moyens algorithmiques de ranger tout cas délictueux possible dans des catégories établies à partir des cas du passé.

Avant d'en venir à l'examen de ce point, il paraît utile de comprendre les raisons qui font que ce désir clairement exprimé par Leibniz persiste aujourd'hui avec tant de vigueur. Il faut, pour ce faire, comprendre ce qui a pu pousser Leibniz à émettre une telle proposition car elle nous renseigne sur les présupposés que l'ambition propre aux Big data tente aujourd'hui de mettre en pratique. 
La proposition exprimée par Leibniz découle de sa méditation sur la nature des caractères de l'alphabet ${ }^{24}$ :

«Or puisque toutes les connaissances humaines se peuvent exprimer par les lettres de l'Alphabet, et qu'on peut dire que celui qui entend parfaitement l'usage de l'alphabet sçait tout; il s'en suit, qu'on pourra calculer le nombre des vérités dont les hommes sont capables <et qu'on peut déterminer la grandeur $>$ d'un ouvrage qui contiendroit toutes les connaissances $<$ humaines $>$ possibles; et ou il y auroit tout ce qui pourroit jamais estre sçû, ecrit, ou inventé; et bien au dela. car il contiendroit non seulement les verités, mais encor les faussetés que les hommes peuvent enoncer; et même des expressions qui ne signifient rien. »

Leibniz estime donc qu'il est possible d'établir que le nombre fini des expressions de la connaissance humaine peut être déterminé avec précision dès lors qu'on retranscrit toute la connaissance au moyen d'un alphabet écrit car l'alphabet est un répertoire fini de caractères (en gros vingt-six lettres dans l'alphabet latin) dont il est possible d'établir le nombre exhaustif de combinaisons, qu'elles soient significatives ou dépourvues de signification. Il est donc possible de dresser l'inventaire de toutes les combinaisons possibles si l'on s'en tient à des assemblages de lettres d'une certaine longueur, que ceux-ci forment ou non des mots ayant un sens. En rappelant le raisonnement suivi par Archimède dans L'arénaire, Leibniz remarque que cet inventaire peut être borné par un maximum. L'existence toujours possible d'un nombre servant de borne supérieure aux combinaisons de l'alphabet rend leur inventaire fini puisqu'il est toujours possible, à chaque moment du temps, d'exhiber un nombre plus grand que le nombre de combinaisons de l'inventaire, même si l'on estime que la connaissance augmente indéfiniment. De là, Leibniz conclut ${ }^{25}$ :

« Mais supposé qu'on aille tousjours en avant < pendant qu'on peut quoyque peut estre lentement pourveu le progres demeure toujours le même, il faut enfin que tout s'epuise $>$ et qu'on ne puisse pas faire de Roman, qu'un autre n'ait deja fait; ny former de chimere nouvelle. Ainsi il faudroit toujours qu'il fut un jour vray au pied de la lettre, qu'on ne dira plus rien, qui n'ait déjà esté dit, nihil dici, quod non dictum sit prius. Car ou l'on dira ce qui a esté dit ou <bien, si l'on veut continuer de dire des choses nouvelles $>$, l'on épuisera ce qui reste encor à dire, $<$ puisque cela

\footnotetext{
24 Leibniz, Opuscules et fragments inédits, ed. E. Couturat, Felix Alcan, Paris, 1903, p. 532.

${ }^{25}$ Leibniz, Opuscules et fragments inédits, ed. E. Couturat, Felix Alcan, Paris, 1903, p. 533.
} 
est fini comme nous demonstrerons tantost. > Il s'agit donc de donner un nombre plus grand que le nombre de tout ce qui se peut dire ou enoncer; c'est ce que nous allons faire. »

Leibniz s'aventure même, dans un autre texte, à opérer le calcul proprement $\operatorname{dit}^{26}$ :

« On peut déterminer le nombre de tous les livres possibles n'excédant pas une longueur finie, formés de mots signifiants ou non-signifiants ; ce nombre comprend aussi par conséquent tous les livres ayant un sens. J'appelle « livre d'une longueur définie » un livre qui ne dépasse pas un nombre déterminé de lettres. [...]. Or le nombre des livres possibles n'excédant pas une telle longueur, qui peuvent être formés d'au plus cent millions de lettres de l'alphabet, est fini. Non seulement ce nombre est fini, mais on peut même trouver par le calcul des combinaisons combien sont possibles de livres, longs ou brefs, différents si peu que ce soit entre eux, n'excédant pas le nombre proposé de lettres. »

Leibniz, dans la reconstruction du texte que propose M. Fichant, en vient donc à établir que le nombre de tous les livres écrits avec cette combinatoire de lettres quel que soit leur sens est de $10^{7300000000000}$ dans lequel un sous-ensemble (que Leibniz ne quantifie pas) seulement sera composé de livres ayant un sens. Leibniz en conclut de façon radicale que, si l'humanité perdure suffisamment longtemps, il n'y aura plus, à un moment donné du temps, que des redites. Le possible de la connaissance est ainsi préempté par un nombre supérieur à toute combinaison de lettres nécessaires pour écrire la connaissance en question et Leibniz en déduit que toute possibilité de connaissance nouvelle n'est en fait qu'une actualisation d'une connaissance passée.

Le raisonnement de Leibniz me semble toutefois critiquable sur au moins trois points. Tout d'abord, il suppose que l'idée de connaissance est fixe une fois pour toutes et qu'elle n'est pas affaire d'interprétation susceptible de reconfigurer dans un sens nouveau tout le savoir accumulé au fil du temps ${ }^{27}$. Dans le cas du droit, cela voudrait dire que la jurisprudence est cumulative et calculable : la jurisprudence n'est pas le témoin d'une modification de la notion de légalité mais seulement l'expansion du domaine de la loi. Mais cette notion de calcul fait problème car elle suppose ensuite

\footnotetext{
${ }^{26}$ Leibniz, De l'horizon de la doctrine humaine (Apokatastasis pantôn), Vrin, Paris, 1991, p. 67.

${ }^{27}$ La connaissance est donc unidirectionnelle et tout est affaire de synthèse. Leibniz l'exprime par exemple sous la forme de l'aphorisme suivant : «Les sciences se résument en s'augmentant ».
} 
que le nombre qui sert de borne supérieure aux combinaisons des caractères ne fait pas partie de la connaissance puisqu'il la « dépasse » mais sans donner à ce nombre de sens proprement dit («dans» la connaissance? « hors» de celle-ci ? Que signifie exactement ce nombre que l'on ne construit pas et qui n'est donc pas objet de connaissance tout en la fondant ?). On ne voit donc pas bien ce que le droit pourrait faire d'une telle notion puisqu'elle est précisément l'écueil de l'ébauche de formalisation de Leibniz. Enfin et surtout, il ne donne aucun moyen de décider entre les combinaisons de caractères porteuses de sens et les autres : que peut bien signifier cette connaissance que l'on n'a pas les moyens de rendre accessible ? La spéculation de Leibniz paraît donc bien hasardeuse mais elle est intéressante parce qu'elle dévoile l'ambition proprement irrationnelle sous-jacente au genre de projets ayant l'alphabet pour raison ultime et dont l'application au cas du droit paraît donc complètement compromise en l'état.

Revenons maintenant au cas des Big data dans le domaine du droit. L'ambition des Big Data aujourd'hui consiste à supposer que les cas juridiques tels qu'ils sont désormais collectés à grande échelle par les différentes instances juridiques seront tous décidables par le biais du calcul : cette ambition est-elle fondée ?

Quelle que soit la réponse technique à cette question, elle repose en fait sur un espoir, en même temps bien éloigné de la philosophie du Leibniz physicien et métaphysicien, mais qui transparaît dans ce projet, formaliste avant la lettre, d'alphabétisation totale de la connaissance : celui d'identifier le possible et le réel, ce qui empêche toute prise en compte d'une virtualité dans les actions humaines, virtualité pourtant indispensable si l'on veut reconnaître la culpabilité d'un individu, c'est-dire sa liberté d'avoir pu faire autrement qu'il n'a fait. On a donc affaire à une conception étroite de la prise de décision qui ne se fait plus en situation d'incertitude du fait de l'énorme réserve de cas que l'on trouve dans les Big data. Cette conception a des origines antiques connues, en particulier dans l'argument dit du « Dominateur ». 


\section{L'argument du Dominateur et la réalité spatio-temporelle du jugement}

L'idée même de légalité suppose, en philosophie du droit (chez Kant par exemple $\left.{ }^{28}\right)$, de faire une distinction entre droit et fait, le premier plan - idéal permettant de juger de la légalité du second - réel. La distinction droit / fait suppose que l'on ait les moyens de penser que ce qui s'est produit aurait pu se produire autrement, c'est-à-dire que ce qui s'est réellement produit aurait pu ne pas se produire du tout : la légalité d'un jugement suppose donc nécessairement l'invocation d'un ensemble de possibilités dont certaines auraient pu ne pas se réaliser. Dans le cas des actes tels qu'ils ont été réalisés, il devient alors possible de déterminer la responsabilité des acteurs puisqu'on pose par principe qu'il s'agit d'individus responsables de leurs actes, c'est-à-dire qu'ils avaient accès dans le passé à un ensemble de possibilités et qu'ils en ont actualisé une par leur choix mais pas celle requise par la loi dans le cas d'un acte considéré comme délictueux. Le jugement consiste donc, dans une première étape, à reconnaître dans cet ensemble de possibilités celle qui a été actualisée et à vérifier si cette actualisation a été ou non faite conformément à la loi.

D'un point de vue logique, l'idée même d'une légalité écarte donc d'entrée de jeu l'«argument du Dominateur » attribué au mégarique Diodore Cronos ${ }^{29}$, argument auquel Aristote s'est par la suite à plusieurs reprises opposé ${ }^{30}$. Comme le rappelle J. Vuillemin, cet argument consiste à dire que :

« Si quelque chose est le cas maintenant, alors il a toujours été vrai que cette chose est ou sera le cas [...]. En conséquent, si l'on accorde que ce qui a été vrai et prévisible de toute éternité ne peut pas ne pas arriver, tout ce qui est en acte est nécessaire ${ }^{31}$

\footnotetext{
28 Kant, Critique de la raison pure, I, 2, "Déduction des concepts purs de l'entendement" : Quand les jurisconsultes parlent de droits et d'usurpations, ils distinguent dans l'affaire la question de droit (quid juris) de la question de fait (quid facti) ; et, comme ils exigent une preuve de chacune d'elles, ils nomment déduction celle qui doit démontrer le droit ou la légitimité de la prétention.

29 Épictète, Entretiens, Livre 2, chap. 19 : « Voici, ce me semble, les éléments dont se compose le sophisme du Dominateur. Il y a incompatibilité entre les trois propositions suivantes : $1^{\circ}$ Tout ce qui est vrai dans le passé est nécessaire; $2^{\circ}$ Possible et impossible sont contradictoires ; $3^{\circ} \mathrm{Il}$ y a du possible qui n'a pas été réalisé et qui ne le sera pas. Diodore, s'apercevant de cette incompatibilité, profita de la vraisemblance des deux premières, pour substituer à la troisième celle-ci : Rien n'est possible que ce qui a été réalisé ou le sera. »

30 Aristote, De l'interprétation, chap. 9. Pour la traduction du texte d'Aristote et son commentaire, voir Vuillemin, J. (1983). "Le chapitre IX du De Interpretatione d'Aristote : Vers une réhabilitation de l'opinion comme connaissance probable des choses contingentes". Philosophiques, 10(1), 15-52. doi:10.7202/203211ar

31 J. Vuillemin, p. 25
} 
L'argument du Dominateur, en partant de la réalité d'un événement ayant eu lieu en conclut donc que sa possibilité était nécessaire d'un point de vue intemporel. Mais ce qui est vrai d'un événement ayant eu lieu vaut également pour tout événement prévisible encore à venir car en effet, comment le statut de réalité d'un même événement pourrait-il changer de modalité quand, contingent dans le futur, il deviendrait nécessaire dans le passé une fois qu'il a eu lieu ? ${ }^{32}$ Pour Diodore, on ne peut donc pas établir de différence entre l'événement possible et l'événement réel sous peine de contradiction concernant la notion même de modalité.

Comment, dans ces conditions, définir la légalité si cette définition suppose la distinction entre différents types de possibilités dont certaines pourraient ne pas s'actualiser, c'est-à-dire dont la puissance d'actualisation n'est pas intégralement décidée à l'avance ?

Comme le remarque encore J. Vuillemin, réussir à envisager l'idéalité du possible autrement que sous la forme d'une totalité indistincte devant nécessairement passer à l'existence en distinguant différents possibles exige de modifier le rapport entre ce qui est exprimé comme une simple possibilité de dicto et ce qui est réalisé dans les faits de re. On doit admettre alors qu'il y a quelque chose d'autre dans le fait tel qu'il s'est réalisé que ce qui en est dit : il y a une nécessité propre à la réalité spatio-temporelle, considérée comme un autre lieu de la nécessité que la pure nécessité intemporelle de la logique stricto sensu. Cette nécessité spécifique relève du domaine de la causalité, c'està-dire de l'enchaînement des événements dans l'espace et le temps selon une nécessité dite seulement « conditionnelle », au sens où elle déploie certes une nécessité interne

\footnotetext{
32 J. Vuillemin p. 25-26 : «En effet, puisque la thèse [de Diodore] est supposée valable pour une chose quelconque quelle que soit la modalité de sa matière, refuser d'appliquer la rétrogradation au futur reviendrait à postuler que seuls les futurs peuvent être contingents ; le monde futur suivrait alors d'autres lois que le monde passé, une supposition d'autant plus ridicule que, la coupure entre passé et futur se déplaçant constamment, le même événement qui n'aurait pas été toujours vrai dans le passé tant qu'il reste futur, le deviendrait dès le moment de son actualisation. S'il peut exister du contingent dans l'avenir, il doit avoir pu exister du contingent dans le passé. »
} 
une fois l'événement commencé mais sans que l'événement soit lui-même conçu comme obligatoire $^{33}$.

Le jugement, dans une deuxième étape, consiste donc à reconnaître l'existence d'une nécessité d'un nouvel ordre dans le passage à l'existence spatio-temporelle qui actualise causalement une simple possibilité. Établir la légalité d'un acte consiste donc désormais à déterminer les raisons qui ont présidé à son passage à l'existence, c'est-àdire à déterminer sa causalité. Un acte relève alors du délictueux quand son passage à l'existence n'a pas été réalisé selon une causalité dont le jugement doit pouvoir déterminer la légitimité. Il est par exemple vain d'en appeler à l'appartenance à un clan familial ou à la vengeance pour justifier un acte criminel, l'appartenance ou la vengeance ne consistant pas une raison nécessaire et suffisante qui justifierait les effets produits par l'acte, considéré de ce fait comme délictueux. Diodore aurait sûrement critiqué cette approche de la légalité qui transforme comme par magie l'idéal en réel en faisant d'un possible contingent un réel nécessaire. Pourtant, cette « magie » existe bel et bien dans l'idée d'une science mathématique de la nature comme dans celle d'une institution juridique, celle du procès. Sans entrer plus en détail dans ce qui distingue ou rapproche ces deux cas, il semble que la forme du jugement dans le cas du procès soit plus immédiatement collective que dans celle de la science dans la mesure où le procès est une institution conçue en vue d'organiser une confrontation entre des voix discordantes, même si la conclusion revient en dernière instance au juge ${ }^{34}$.

Si l'on s'en tient donc au cas de l'expérience de la légalité juridique, déterminer si un acte est ou non délictueux s'opère par l'intermédiaire de l'institution du procès envisagé comme le lieu de synthèse de deux nécessités, possible et causale. Ainsi, à

\footnotetext{
33 J. Vuillemin p. 29: «Les exemples qu'Ammonius donne de la nécessité simple : « les angles d'un triangle ont leur somme égale à deux droits », « le Ciel se meut toujours », « le feu est chaud», « Socrate est mortel» expriment des cas de prédication essentielle, dans lesquels l'appartenance nécessaire du prédicat au sujet est, soit éternelle, soit coextensive à la durée du sujet. Les énoncés : 《le soleil est occulté par la lune », «Socrate marche » fournissent des exemples de nécessité conditionnelle, dans lesquels l'appartenance du prédicat au sujet n'est nécessaire que durant l'actualité de l'événement : occultation ou marche. »

34 La différence entre l'aspect collectif dans la science et dans le droit pourrait aisément se discuter, les institutions rendant la science possible n'étant pas moins collectives que celles qui relèvent du droit, même si elles sont moins directement visibles.
} 
partir du même fait réalisé, Diodore en conclut que le possible était déjà réel de toute éternité tandis qu'un procès suppose au contraire que l'événement réel n'était finalement qu'un possible qui n'était pas nécessairement actualisable. Le procès doit donc commencer par établir que l'acte délictueux n'était pas une nécessité simple (étape $\mathrm{n}^{\circ} 1$ ) parce que sa causalité reste contingente (étape $\mathrm{n}^{\circ} 2$ ) et que par conséquent, il est possible, dans une certaine mesure qui reste à définir par le jugement, de dépouiller l'acte délictueux des effets qu'il a eus en réaffirmant sa virtualité. Déterminer cette mesure consiste à parvenir à faire que les effets dans le présent de l'acte reconnu comme délictueux soient rapportés seulement à un possible. Cette rétroaction est l'objet d'un travail intersubjectif sur le sens de l'action parce qu'un possible n'est pas isolé : il s'agit d'un point de vue impliquant qu'il y en ait d'autres, ce qui suppose du sens partagé qui ne soit pas nécessairement convergeant et fasse l'objet d'un débat argumenté. Le jugement consiste donc à insuffler collectivement, dans l'irréversibilité unique de ce qui ne peut plus être modifié (les effets d'une acte considéré comme délictueux), un halo de virtualité qui permette à l'acte d'être remis collectivement en perspective, c'est-àdire, assez curieusement au premier abord, d'être rendu au possible. Le possible n'est pas conçu ici comme chronologiquement antérieur au réel, il se situe dans une autre dimension que l'acte réel, celle qui a trait au sens tel qu'il est collectivement conçu.

Ainsi, pour résumer, alors que le jugement vise à établir que « hier ne sera pas semblable à demain », le point de vue d'un Diodore relayé aujourd'hui par les données massives serait de dire à l'inverse : «demain sera semblable à hier ». Diodore critiquerait donc toute nouveauté du futur en disant que cela revient à nier les faits en envisageant le réel comme un simple possible, c'est-à-dire en modifiant sa modalité, nécessairement univoque. À l'opposé, on pourrait objecter à Diodore que, tout rigoureux qu'il soit du point de vue de la modalité logique, il est tout simplement victime d'un usage abusif de la figure rhétorique de la prolepse dans laquelle on fait comme si demain, conçu comme simple possibilité, était déjà réalisé aujourd'hui comme 
réalité. L'opposition des deux points de vue - celui d'un Diodore conçu comme « proleptique » et celui du jugement collectif conçu comme « virtualisant »-, loin d'être dépassée, revient en force aujourd'hui dans le cadre de la digitalisation juridique. Elle a pour origine l'ambivalence du concept de calcul qu'il faut réussir prendre en compte dans sa globalité pour rendre le concept de volonté à sa destination première : permettre le jugement.

\section{Le jugement, à la croisée du calculable et de l'incalculable}

L'introduction de moyens informatiques au service du droit et de la justice n'est pas en soi une hérésie si l'on y voit une nouvelle façon de penser les rapports entre deux niveaux, spatio-temporel d'une part et graphique-logique de l'autre, nécessaire à l'élaboration d'un jugement de droit. Cette introduction permet de s'adapter au fait que les interactions sociales, parce qu'elles sont aujourd'hui pour la plupart informatiquement médiatisées, produisent massivement des données, c'est-à-dire des traces graphiques qui demandent à être constamment agrégées par le biais de logiciels en vue d'être ultérieurement interprétées. Si les deux dimensions du concept de calculabilité sont prises ensemble en considération, il devient possible de sortir du cercle vicieux qui consiste, en privilégiant une dimension sur l'autre, à détruire la capacité de juger et de reconnaître alors pleinement le caractère à jamais ouvert de tout jugement. 


\section{Bibliographie}

F. Bailly et G. Longo, Mathématiques et sciences de la nature ; la singularité physique du vivant, Hermann, Paris, 2007.

L. Couturat, La logique de Leibniz, Alcan, Paris, 1901.

R. Descartes,

- Discours de la méthode, AT VI

[https://fr.wikisource.org/wiki/Discours_de_la_m\%C3\%A9thode/\%C3\%89dition_A dam_et_Tannery] Paris

- Méditations, AT IX-1

[https://fr.wikisource.org/wiki/Livre:Descartes_-

_\%C5\%92uvres,_\%C3\%A9d._Adam_et_Tannery,_IX.djvu]

Épictète, Entretiens, Paris, Gallimard, 1993.

G. Galilée, L'essayeur, Presses universitaires de Franche-Comté, 1989.

A. Garapon et J. Lassègue, Justice digitale, Paris, PUF, 2018.

E. Kant, Critique de la raison pure, Paris, PUF, 2012.

R. Lachterman, The Ethics of Geometry: a Genealogy of Modernity, Routledge, Londres, 1989.

J. Lassègue \& G. Longo (2012), "What is Turing's Comparison between Mechanism and Writing Worth?" dans S.B. Cooper, A. Dawar, and B. Löwe (Eds.), How the World Computes, Lecture Notes in Computer Science 7318, Springer: 451-462.

J. Lassègue (2002), "Turing, entre formel et forme ; remarques sur la convergence des perspectives morphologiques", Intellectica, 2002/2 (35): 185-198

G. W. F. Leibniz,

"Nova Methodus pro Maximis et Minimis", dans La naissance du calcul différentiel :

26 articles des Acta Eruditorum, Paris, Vrin, 1995.

"De Justitia et Novo Codice" (1678), Philosophische Schriften, Leibniz Forschungstelle Universität Münster, Band IV, 1999.

"Dialogus", die Philosophischen Schriften, Gerhardt ed., Band VII, 1875. 
- Opuscules et fragments inédits, L. Couturat ed., Felix Alcan, Paris, 1903, p. 532.

- De l'horizon de la doctrine humaine (Apokatastasis pantôn), Paris, Vrin, 1991,

L. Lessig, "Code is Law" (2000), dans Code v.2.0, New York, Perseus, 2006.

[http://codev2.cc]

G. Longo,

- "Laplace, Turing et la géométrie impossible du « jeu de l'imitation »: aléas, déterminisme et programmes dans le test de Turing" (2002), Intellectica, n. 35, 2002/2, pp. 131-162.

- "Au sujet des lois dans les sciences humaines et de la nature" (2017) dans Loi des hommes des dieux et de la nature ; éléments pour une analyse transversale, Paris, Spartacus-IDH, 2017.

Giuseppe Longo et Maël Montévil (2013), "Extended criticality, phase spaces and enablement in biology" dans Chaos, Solitons and Fractals, Elsevier, 2013, Emergent Critical Brain Dynamics, 55, pp.64-79.

[hal-01192911v2]

Giuseppe Longo, Maël Montévil et Arnaud Pocheville (2014), "L'incompressible complexité du réel et la construction évolutive du simple", dans Complexité-Simplexité, A. Berthoz et J.-L. Petit eds, Paris, Collège de France, 2014.

[http://books.openedition.org/cdf/3363]

Mate Paksy, "Leibniz et les questions de l'ontologie juridique : la science, les règles et le concept du droit" (2018), Astérion, 19 | 2018.

[http://journals.openedition.org/asterion/3573 ; DOI : 10.4000/asterion.3573]

A. Rouvroy \& B. Stiegler (2015), « Le régime de vérité numérique », Socio, 4 | 2015, 113-140.

A. M. Turing (1936), "On Computable Numbers with an Application to the Entscheidungsproblem", Proceedings of the London Mathematical Society, Volumes 242, Issue 1, 1 January 1937: 230-265.

J. Vuillemin, (1983). "Le chapitre IX du De Interpretatione d'Aristote : Vers une réhabilitation de l'opinion comme connaissance probable des choses contingentes". Philosophiques, 10(1), 15-52. 